\title{
Partition functions and equilibrium constants for diatomic molecules and atoms of astrophysical interest ${ }^{\star}$
}

\author{
P. S. Barklem ${ }^{1}$ and R. Collet ${ }^{2,3}$ \\ 1 Theoretical Astrophysics, Department of Physics and Astronomy, Uppsala University, Box 516, 75120 Uppsala, Sweden
e-mail: Paul. Barklemaphysics.uu.se
2 Research School of Astronomy and Astrophysics, Australian National University, Canberra, ACT 2611, Australia
3 Stellar Astrophysics Centre, Department of Physics and Astronomy, Aarhus University, Ny Munkegade 120, 8000 Aarhus C,
Denmark
}

Received 14 July 2015 / Accepted 28 January 2016

\section{ABSTRACT}

\begin{abstract}
Partition functions and dissociation equilibrium constants are presented for 291 diatomic molecules for temperatures in the range from near absolute zero to $10000 \mathrm{~K}$, thus providing data for many diatomic molecules of astrophysical interest at low temperature. The calculations are based on molecular spectroscopic data from the book of Huber \& Herzberg (1979, Constants of Diatomic Molecules) with significant improvements from the literature, especially updated data for ground states of many of the most important molecules by Irikura (2007, J. Phys. Chem. Ref. Data, 36, 389). Dissociation energies are collated from compilations of experimental and theoretical values. Partition functions for 284 species of atoms for all elements from $\mathrm{H}$ to $\mathrm{U}$ are also presented based on data collected at NIST. The calculated data are expected to be useful for modelling a range of low density astrophysical environments, especially star-forming regions, protoplanetary disks, the interstellar medium, and planetary and cool stellar atmospheres. The input data, which will be made available electronically, also provides a possible foundation for future improvement by the community.
\end{abstract}

Key words. molecular data - atomic data

\section{Introduction}

Calculations of the relative abundances of molecules and atoms are required for the modelling of a wide range of cool astronomical objects and their spectra, e.g. the solar atmosphere, sunspots, cool stellar atmospheres, exoplanetary atmospheres and comets; for a recent review see Bernath (2009). In 1979, Huber and Herzberg published their classic compilation of constants for diatomic molecules (Huber \& Herzberg 1979, hereafter HH). Soon after, Sauval \& Tatum (1984, hereafter ST) published a set of partition functions and equilibrium constants for 301 diatomic molecules of possible astrophysical interest based on data from this compilation. They calculated for temperatures in the range from 1000 to $9000 \mathrm{~K}$, arguing that molecular equilibrium will not be valid in astrophysical objects at lower temperatures. Nevertheless, molecular equilibrium is still often useful, either to use directly in exploratory models, or as an initial guess for non-equilibrium modelling. For example, Bond et al. (2010) have modelled terrestrial planet formation assuming chemical equilibrium, supported by arguments regarding the relevant timescales for planetesimal formation and observational evidence from our solar system. In this work we redo the calculations of ST, with the main goal to extend the temperature range of the calculations down to temperatures near absolute zero through removal of various high-temperature approximations related to the rotational and nuclear hyperfine structure levels.

* Full Tables 1-8 are only available at the CDS via anonymous ftp to cdsarc.u-strasbg.fr (130.79.128.5) or via

http://cdsarc.u-strasbg.fr/viz-bin/qcat?J/A+A/588/A96
These calculations allow us to make some additional improvements. First, since the HH data is now made available electronically via the NIST $^{1}$ Chemistry Webbook (Huber et al. 2011), all states and coefficients could be included with little effort. Second, critically compiling input molecular data is a complex problem, one an expert in molecular physics could spend a career on: Huber and Herzberg spent over 30 years making their compilation. Thus, ST chose to adopt data exclusively from $\mathrm{HH}$ so that there could be no ambiguity regarding what data was used, even though this meant that improvements from after $\mathrm{HH}$ finalised data for a given molecule were omitted. This problem can now be relatively easily circumvented by making the input data files available electronically, allowing data sets to be merged in a transparent fashion. Finally, the possibility to provide large tables online means results can be presented for a fine temperature grid and removes the need for polynomial representations.

There have been countless improvements and additions to known molecular data during the over 30 years since HH's compilation. The dissociation energy is of particular importance in the calculation of the equilibrium constant, and so in this work we have compiled updated dissociation energies based on two main sources: the compilation of experimental bond energies of Luo (2007), and the theoretical calculations of Curtiss et al. (1991) using Gaussian-2 theory. Regarding spectroscopic constants, the most significant update was the inclusion of constants for the ground states of 85 of the most important molecules according to the compilation by Irikura (2007); this addition is

\footnotetext{
1 National Institute of Standards and Technology, http://www . nist.gov/
} 
of particular importance in updating the $\mathrm{HH}$ data for this work. Further, in the case of a few molecules of particular astrophysical interest for which data did not previously exist (e.g. FeH, $\mathrm{TiH}$ ) or improved data has now become available (e.g. $\mathrm{CrH}$, $\mathrm{ZrO}$ ), data were added or updated, respectively. We emphasise that we have not attempted here to make a comprehensive review of all 291 molecules; however, the input data presented here provides a possible foundation for workers to be able to incorporate further improvements for specific molecules of particular interest. Our primary goal here is to provide a comprehensive data set for a large number of molecules suitable for astrophysical modelling, using up-to-date, or at least reasonably close to up-to-date, data in the most important cases. A few molecules were also removed from the list of ST, and the final list contains 291 diatomic molecules; all improvements and removals with respect to ST, are detailed in Sect. 2.1.2.

In order to calculate equilibrium constants, in addition to partition functions for the molecule, partition functions for the component atoms are needed. These partition functions are of considerable interest in themselves, and have also been calculated based on data from NIST for all chemical elements from hydrogen to uranium, for neutral and singly- and doubly-ionised species.

The paper is structured as follows. In Sect. 2 the calculations and the input data are described in detail. In Sect. 3 the results are analysed and compared with previous results.

\section{Calculations}

The expressions used for calculating the partition function corresponds to those from (Irwin 1987, Eqs. (26)-(31)), though neglecting spin-orbit coupling. We write the partition function as:

$$
\begin{aligned}
Q=\sum_{e} & \sum_{v=0}^{v_{\max }} \sum_{J=\Lambda}^{J_{\max }} g_{\Lambda, \mathrm{hfs}}(2 S+1)(2 J+1) \\
& \times \exp \left[-\frac{h c}{k T}\left(T_{e}+G(v)+F_{v}(J)-E_{0}\right)\right],
\end{aligned}
$$

where $e$ denotes electronic states with projection of orbital angular momentum quantum number $\Lambda$ and total spin quantum number $S$, and $v$ and $J$ are the vibrational and rotational quantum numbers, respectively. $T_{e}, G$ and $F$ represent the electronic, vibrational and rotational energies in wavenumber units. $E_{0}$ is the energy of the lowest state of the molecule (i.e. $v=0, J=\Lambda$ for the ground electronic state); we note that although this term can be taken outside the summation, at low temperatures it is best left in the summation to avoid numerical errors.

The vibrational and rotational energies can be expressed as asymptotic expansions in terms of molecular constants. In this work we use the usual band spectrum constants as defined in Herzberg (1950) and used by HH. The vibrational energy is

$G(v)=w_{e}\left(v+\frac{1}{2}\right)-w_{e} x_{e}\left(v+\frac{1}{2}\right)^{2}+w_{e} y_{e}\left(v+\frac{1}{2}\right)^{3}$.

The rotational energy is

$F_{v}(N)=B_{v} J(J+1)-D_{v}(J(J+1))^{2}$,

where

$B_{v}=B_{e}-\alpha_{e}\left(v+\frac{1}{2}\right)+\gamma_{e}\left(v+\frac{1}{2}\right)^{2}$, with the centrifugal distortion term

$D_{v}=D_{e}-\beta_{e}\left(v+\frac{1}{2}\right)$.

The statistical weight factor relating to $\Lambda$-doubling and nuclear hyperfine structure $g_{\Lambda, \mathrm{hfs}}$ is calculated according to Irwin (1987, Table 3 and Eqs. (28) and (29)). Note that our calculations follow the usual convention that the statistical weight is divided by the product of the nuclear spin statistical weights, i.e. nuclear spin parts are not included.

The upper limit $v_{\max }$ for summation over vibrational quantum number $v$ in Eq. (1), is found naturally by treating Eq. (2) as an asymptotic expansion. Normally, the best approximation is achieved when the asymptotic expansions are truncated at the smallest term. Due to the small number of terms available, to ensure the convergence of the included terms we used a slightly more stringent condition: to be included in the summation a term should not be more than half of the preceding term. In cases where $w_{e} x_{e}$ is not available or able to be estimated, the summation is arbitrarily truncated at $v_{\max }=100$. For the summation over rotational quantum number $J$, the value of $J$ with the largest thermal population can be easily calculated (see p. 124 of Herzberg 1950). As the population decreases exponentially at larger $J$, we chose to truncate the summation at $J_{\max }$ equal to 10 times this value, but with a minimum value of 10 , which is used at low temperatures when $J=0$ is the most populated state (for $\Sigma$ states).

The main differences between our calculations and those of ST are: a) we have not employed the high-temperature approximation for the summation over rotational quantum number $J$; b) we have not used the approximate expression for the statistical weight $g$; c) we include higher order terms in the asymptotic expansions if available. Both a) and b) are necessary to extend the calculations to low temperatures, as both these approximations are only valid in the high-temperature case where many rotational and nuclear hyperfine levels contribute to the partition function.

As mentioned earlier, in order to compute the molecular equilibrium constants, we need to evaluate partition functions for the relevant atoms and ions as well. We calculate them using the following expression:

$Q_{\text {atom }}=\sum_{i}\left(2 J_{i}+1\right) \mathrm{e}^{-\chi_{i} / k T}$

where the index $i$ refers to the atomic energy level, $J_{i}$ is the angular momentum of the level and $\chi_{i}$ its excitation energy relative to the ground state. In the calculations, we account explicitly for fine and hyperfine structure of atomic levels and again follow the usual convention that the nuclear spin statistical weights are not included.

For isolated atoms and molecules the partition function formally diverges due to an infinite number of Rydberg states approaching the ionisation threshold. However, in real physical environments the sum is modified due interactions of nearby particles. This may be viewed in the so-called "physical" or "chemical" pictures (e.g. Däppen et al. 1987; Rogers et al. 1996). In the physical picture, nearby particles modify the electronic potential from the spherically symmetric Coulomb potential, thus resulting in a finite number of states. In the chemical picture, one begins with the isolated atoms and molecules and account for loosely bound states being "dissolved" into continuum states if a perturbing particle is sufficiently nearby. Inclusion of these effects would lead to partition functions and equilibrium constants dependent on densities of the most common perturbers 
Table 1. Sample of table containing dissociation energies from the literature, and final adopted values.

\begin{tabular}{|c|c|c|c|c|c|c|c|c|c|c|}
\hline \multirow{2}{*}{\multicolumn{3}{|c|}{$\begin{array}{c}\text { Dissociation energies } \\
\text { Molecule }\end{array}$}} & \multirow{2}{*}{\multicolumn{2}{|c|}{$\begin{array}{c}291 \text { molecules } \\
\text { HH }\end{array}$}} & \multirow{2}{*}{\multicolumn{2}{|c|}{ Luo }} & \multirow{2}{*}{\multicolumn{2}{|c|}{ G2 }} & \multirow{2}{*}{\multicolumn{2}{|c|}{ Adopted }} \\
\hline & & & & & & & & & & \\
\hline & & & & & & & & & & \\
\hline $\mathrm{H} 2$ & $\mathrm{H}$ & $\mathrm{H}$ & 4478130 & & 4.478007 & 0000004 & & & 4478007 & 0000004 \\
\hline $\mathrm{Li} 2$ & $\mathrm{Li}$ & $\mathrm{Li}$ & 1.046000 & . & 1.049900 & & 1.124000 & . & 1.049900 & \\
\hline B2 & B & B & 3.020000 & . & 2.802000 & & & . & 2.802000 & \\
\hline $\mathrm{C} 2$ & $\mathrm{C}$ & $\mathrm{C}$ & 6.210000 & . & 6.371000 & 0.160000 & 6.401000 & . & 6.371000 & 0.160000 \\
\hline N2 & $\mathrm{N}$ & $\mathrm{N}$ & 9.759400 & . & 9.753940 & 0.000900 & 9.705000 & . & 9.753940 & 0.000900 \\
\hline $\mathrm{O} 2$ & $\mathrm{O}$ & $\mathrm{O}$ & 5.115600 & . & 5.116420 & 0.000130 & 5.013000 & . & 5.116420 & 0.000130 \\
\hline $\mathrm{F} 2$ & $\mathrm{~F}$ & $\mathrm{~F}$ & 1.602000 & . & 1.605960 & 0.001000 & 1.587000 & . & 1.605960 & 0.001000 \\
\hline
\end{tabular}

Notes. The full table is available at the CDS.

(hydrogen atoms, ions, electrons, etc.), leading to significantly increased complexity, and perhaps limiting the usefulness, of the results. In these calculations we have included all available states from the relevant sources, without modification, and will show in Sect. 3 that this leads to only small errors for typical low density astrophysical environments where this data might be applied, such as stellar atmospheres.

Once partition functions for the molecule and constituent atoms and ions are known, the equilibrium constant $K_{\mathrm{AB}}$ can be easily calculated. In terms of partial pressures (e.g. Tatum 1966)

${ }^{p} K_{\mathrm{AB}}=\frac{p_{\mathrm{A}} p_{\mathrm{B}}}{p_{\mathrm{AB}}}=\left(\frac{2 \pi m k T}{h^{2}}\right)^{3 / 2} k T \frac{Q_{\mathrm{A}} Q_{\mathrm{B}}}{Q_{\mathrm{AB}}} \mathrm{e}^{-D_{0}^{0} / k T}$.

The partition function and equilibrium constant calculations were carried out as detailed above using a computer program written in IDL.

\subsection{Input molecular data}

The input data for the molecular partition function calculations consists of molecular terms containing relevant quantum numbers $\Lambda$ and $S$ and spectroscopic constants $T_{e}, w_{e}, w_{e} x_{e}, w_{e} y_{e}, B_{e}, \alpha_{e}, \gamma_{e}, D_{e}, \beta_{e}$. In the homonuclear case, parity (u or g) and reflection symmetry (+ or - ), as well as the nuclear spins for the component atoms, $I_{1}$ and $I_{2}$, are also needed in the calculation of $g_{\Lambda, \mathrm{hfs}}$. Calculation of the equilibrium constant requires the dissociation energy $D_{0}^{0}$. Below we detail the data used in our calculations.

\subsubsection{Dissociation energies}

The exponential term in Eq. (7) containing the dissociation energy $D_{0}^{0}$ arises since the atomic and molecular partition functions are calculated with reference to different zero points, their respective ground states. The exponential dependence on $D_{0}^{0}$ means $K_{\mathrm{AB}}$, and thus the partial pressure $p_{\mathrm{AB}}$, number density $n_{\mathrm{AB}}$ and opacity $\kappa_{\mathrm{AB}}$, in an astrophysical model, are sensitive to errors in $D_{0}^{0}$. The relative error from this source in the partial pressure or number density of the molecule, and thus its opacity $\kappa_{\mathrm{AB}}$, is given by

$\frac{\Delta n_{\mathrm{AB}}}{n_{\mathrm{AB}}}=\frac{\Delta p_{\mathrm{AB}}}{p_{\mathrm{AB}}}=\frac{\Delta \kappa_{\mathrm{AB}}}{\kappa_{\mathrm{AB}}}=\exp \left(\Delta D_{0}^{0} / k T\right)-1$.

For example, at $3500 \mathrm{~K} k T \sim 0.3 \mathrm{eV}$ and so $\Delta D_{0}^{0} \sim 0.1 \mathrm{eV}$ leads to errors of around $40 \%$. The error increases as temperature decreases; for example at $1000 \mathrm{~K}$, the same error in $D_{0}^{0}$ leads to an error in excess of a factor of 2.

Thus, it is important to have accurate dissociation energies, and we made a significant effort to collect updated data. Data were collected from three sources: HH, Luo (2007), and Curtiss et al. (1991). The data from the compilation of $\mathrm{HH}$ is compiled as a starting reference point, but is rarely adopted (only for $\mathrm{HeH}^{+}$, $\mathrm{NeH}^{+}, \mathrm{HF}^{+}$). Luo (2007) presents a comprehensive survey of experimental chemical bond energies, from which $D_{0}^{0}$ can be easily calculated. For each molecule, Luo (2007) presents a survey of available data, and often recommends one of the values. If a recommended or only a single value is available, then this is was the value collected. If no value was recommended we collected the value with the smallest estimated uncertainty. Finally, data from the theoretical calculations of Curtiss et al. (1991) were also collected. This work employs so-called Gaussian-2 theory, essentially ab initio molecular structure calculations using Gaussian orbitals; see Curtiss et al. (1991).

We then evaluated the data in each case and adopted a final value. In the vast majority of cases, the value from Luo (2007) was adopted. The Luo (2007) survey usually includes the experimental data in $\mathrm{HH}$ together with error estimates. As error estimates were rarely given by $\mathrm{HH}$, Luo (2007) is always preferred over $\mathrm{HH}$ even if the data are from the same original source. In the majority of cases, the Luo (2007) values and the Curtiss et al. (1991) values agreed well, and where this was the case we favoured the Luo (2007) values as they are experimental and have error estimates. However, in cases where disagreement was substantial, and the available experimental data in Luo (2007) were deemed uncertain, we have favoured the theoretical values from Curtiss et al. (1991) (see discussion in Costes \& Naulin 1994).

Data from the three different sources and the adopted data are presented in Table 1. Comparisons of the data, where they overlap, are given in Fig. 1. We see that the correlation is generally good between the three sources, yet the scatter is generally of order a few $0.1 \mathrm{eV}$, and some cases disagreeing by 1 or even $2 \mathrm{eV}$. Figure 2 compares the final adopted values with those from $\mathrm{HH}$. We see rather large changes in molecules such as $\mathrm{H}_{2}^{-}$(which we note is still rather uncertain), $\mathrm{MgO}$ (which was noted by Costes \& Naulin 1994), $\mathrm{CaO}, \mathrm{NH}^{+}, \mathrm{NS}^{+}$and $\mathrm{C}_{2}^{+}$, just to pick out some of the largest with constituent atoms that are astrophysically abundant. 

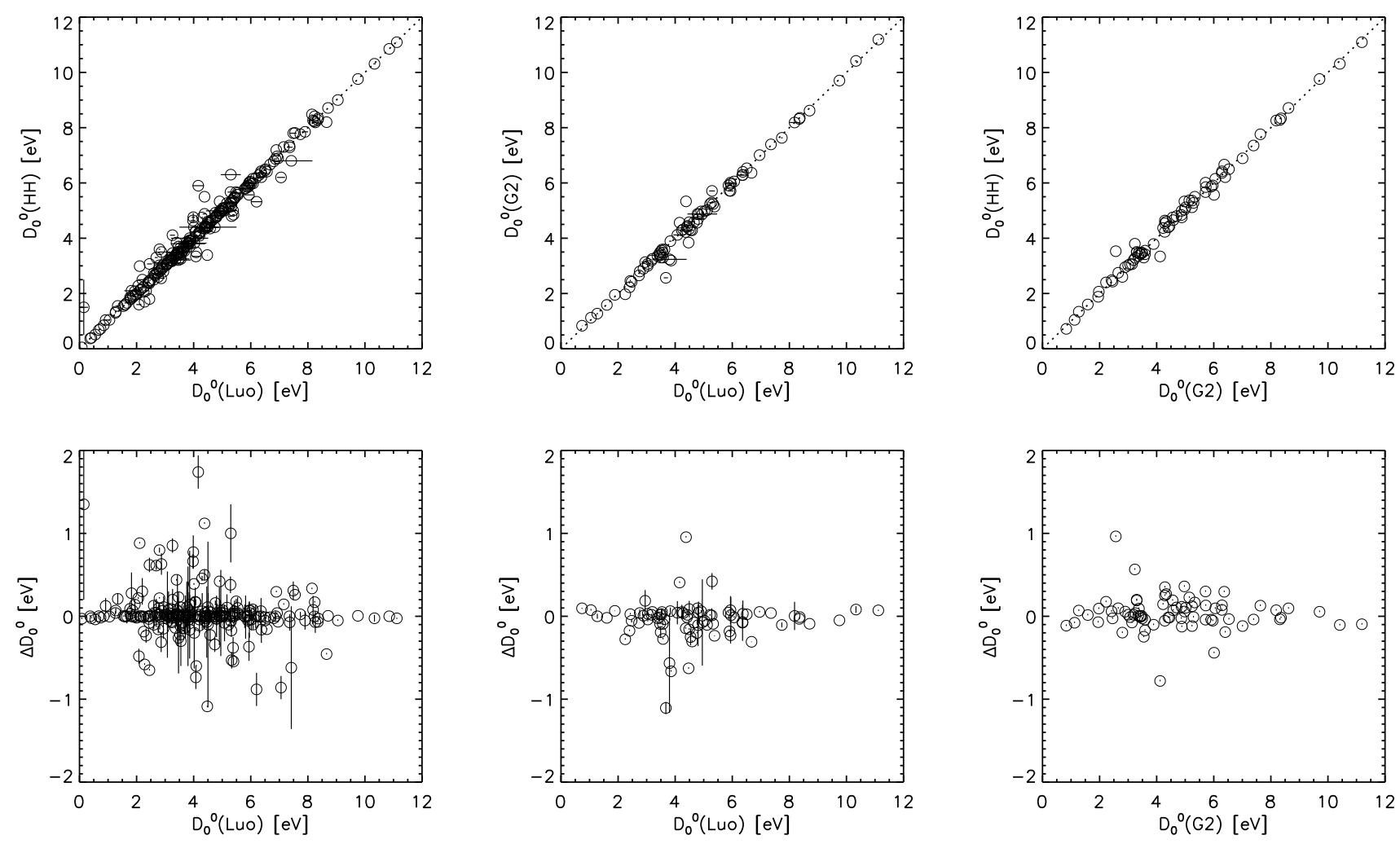

Fig. 1. Comparison of dissociation energies $D_{0}^{0}$ from the three main sources discussed in the text. The bars show estimated errors where available. The theoretical values of Curtiss et al. (1991) are labelled G2, denoting Gaussian-2 theory. The difference shown in the lower panel is always in the sense ( $y$-axis $-x$-axis). The error shown in the difference is taken as the sum of the errors, where available.

\subsubsection{Spectroscopic constants}

The starting point for our calculations are the molecular constant data from $\mathrm{HH}$, which were extracted electronically from the NIST Chemistry WebBook (Huber et al. 2011, retrieved October 14, 2011) for all molecules considered by ST. An IDL computer program was then used to automatically extract the required data from these html files. Changes and additions to the data are then made by merging the data with extra data sets, also with an IDL computer program. The most important of these changes and additions is the compilation of updated constants for the ground states of 85 molecules by Irikura (2007, 2009). Further changes and additions were also made as listed below. Note, for negative ions we followed the treatment of ST (see Sect. $2 \mathrm{a}$ of that paper) adopting estimates of data and ground state designations from similar molecules where necessary.

Additional molecules. $\mathrm{TiH}$ and $\mathrm{FeH}$ were added using data from Burrows et al. (2005) and Dulick et al. (2003), respectively. $\mathrm{CH}^{-}$was also added, which is not present in Table 6 of ST despite being mentioned in Sect. $2 \mathrm{a}$ of that paper. In this case we took the molecular term for the ground state from $\mathrm{HH}$, and used spectroscopic constants from the $\mathrm{CH}$ ground state.

Updates to data. The constants for $\mathrm{CrH}$ have been updated using data from Bauschlicher et al. (2001, Table II), and from Ghigo et al. (2004) for higher states, prefering CASPT2 calculations. The energy levels for $\mathrm{ZrO}$ have been updated following Davis \& Hammer (1988). The constants for the ground state of $\mathrm{NeH}^{+}$have been taken from Civiš et al. (2004).
Estimates. Data are missing for some states with identified quantum numbers and electronic energies $T_{e}$, and so rather than omit these states, in certain cases we chose to make simple estimates based on data from other states of the molecule or similar molecules. In cases where $w_{e}$ or $B_{e}$ is missing, but available for other states of the molecule, we adopted the average of the values for the other states. In cases where $w_{e}$ or $B_{e}$ is missing for the ground state of a molecule that has no other states with these values, then an estimate was taken from a similar molecule (e.g. oxide or homo-nuclear molecule with nearest mass) where data is available for the ground state.

Removed molecules. Some molecules included in ST, were not included in this work. $\mathrm{PdH}, \mathrm{ClF}, \mathrm{AuSi}, \mathrm{CuSe}$ were missing in the NIST Webbook and not deemed important enough to warrant followup. $\mathrm{HeH}^{+}, \mathrm{CH}^{+}$and $\mathrm{LaO}$ were also missing, but included using data from $\mathrm{HH}$ added by hand. $\mathrm{LaH}, \mathrm{BH}^{+}, \mathrm{RuO}, \mathrm{AgO}, \mathrm{CeO}$, $\mathrm{IrO}, \mathrm{FeF}, \mathrm{MnS}, \mathrm{FeCl}$ either do not have an identified ground state or do not have data for the ground state and were not deemed important enough to warrant followup. It seems that ST assumed a ground state for these cases. For $\mathrm{CN}^{+}$the ground state is also not identified in $\mathrm{HH}$, but due to its possible astrophysical importance we chose in this case to assume that the lowest known state, $a^{1} \Sigma$, was the ground state. We note the inherent danger in such a procedure: e.g., the ground state of $\mathrm{FeCl}$ is uncertain in $\mathrm{HH}$, and later work by Delaval et al. (1980) showed it to be a state with different properties to those identified as candidates in $\mathrm{HH}$.

The final input data for the calculations are written to files containing all relevant information, including dissociation energies, nuclear spins, and spectroscopic constants. Labels 

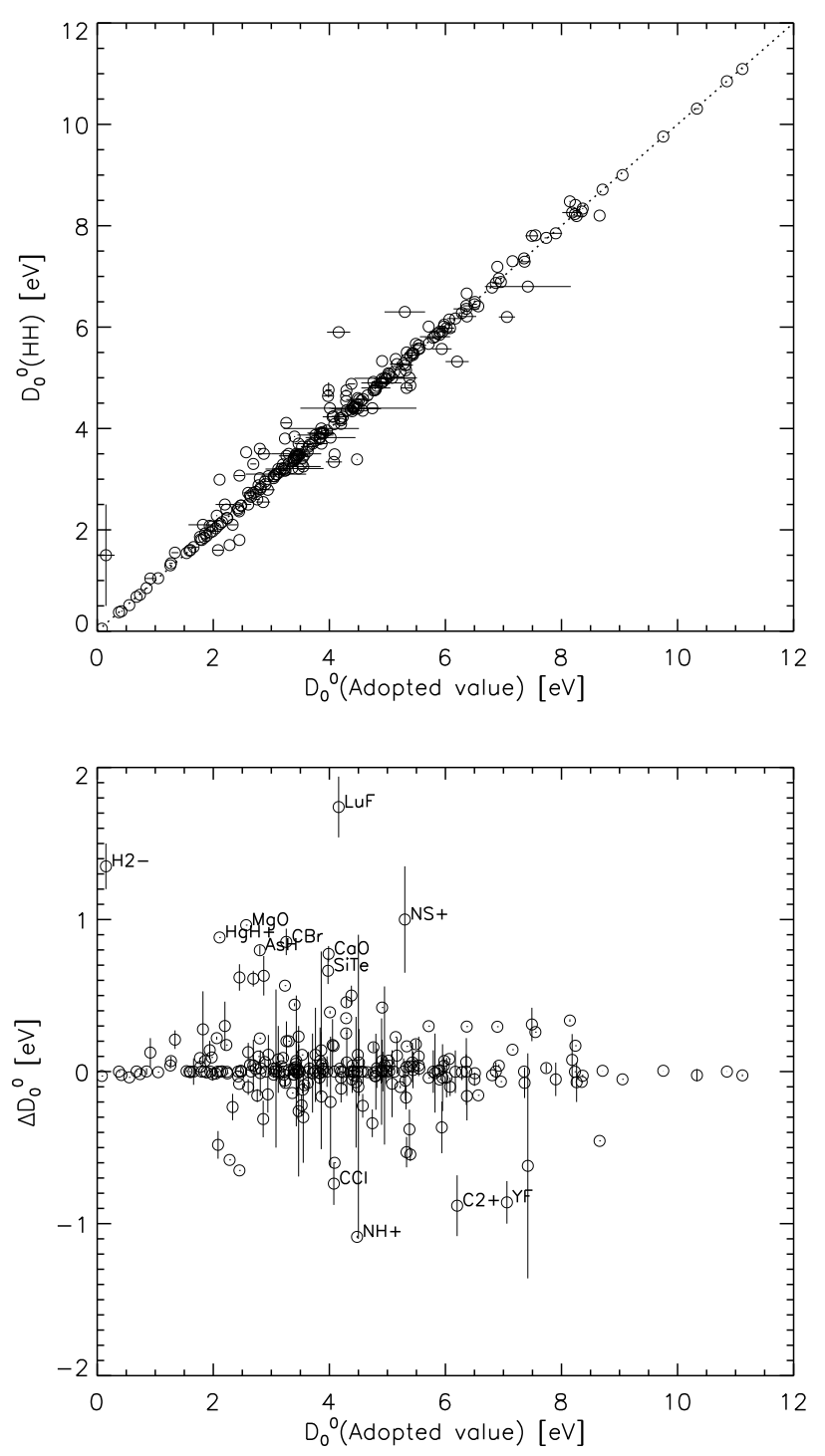

Fig. 2. Comparison of final adopted values of the dissociation energies $D_{0}^{0}$ with those of $\mathrm{HH}$. The bars show estimated errors where available. The difference is in the sense (HH - adopted). The error shown in the difference is taken as error in the adopted value, where available. The cases with the largest differences are labelled.

indicating the source of the data are included for all spectroscopic constants. These files are available from the CDS. A sample of an example file for $\mathrm{H}_{2}$ is given in Table 2. The key to the reference labels used in these files is given in Table 3. We note that states where fine structure components are given in $\mathrm{HH}$, with corresponding projection of total angular momentum quantum number $\Omega$, the value of the spin multiplicity $2 S+1$ is adjusted to unity in order to achieve the correct statistical weight.

\subsection{Input atomic data}

The input data for the atomic partition function consists of angular momenta and excitation energies of energy levels of atoms and ions for all elements between $\mathrm{H}$ and $\mathrm{U}$ and ionisation stages from I to III (neutral and singly and doubly ionised). The information was extracted electronically from the NIST atomic spectra database (Kramida et al. 2014), which includes data about atomic levels collected and critically assembled from various compilations. The complete list of references for the atomic energy level information is provided as electronic material (a sample of which is given in Table 5). Partition functions for atoms and ions are then computed by simply combining the data according to Eq. (6).

Ionisation energies for individual atoms and ions are not strictly relevant for the calculations of atomic partition functions; however, knowing their values is necessary in order to compute ionisation equilibria. For completeness and for the user's convenience, we have therefore elected to compile and make available electronically a table of ionisation energies for all elements between $\mathrm{H}$ and $\mathrm{U}$. The ionisation energy data comes primarily from the CRC Handbook of Chemistry and Physics (Haynes 2010); data missing from the primary source or affected by large uncertainties (mainly ionised stages of very heavy elements) have been filled in using theoretical or numerically interpolated values extracted from the NIST database (Kramida et al. 2014). A sample of the table is shown in Table 4.

\section{Results and discussion}

To present the final calculations we must first decide on a temperature grid for the final calculations. This should ideally be as sparse as possible for presentation reasons, while retaining a sufficient number of points for precise use in applications. To decide the temperature grid we first made a calculation on a fine logarithmic grid of 101 points ranging from 0.1 to $10^{4} \mathrm{~K}$. In implementing the results in a spectrum synthesis code, Jeff Valenti (priv. comm.) calculated an adaptive grid that, when used with cubic spline interpolation in $\log _{10}(Q)$ for partition functions and in $\log _{10}\left({ }^{p} K\right)+\left(D_{0} \times 5040 / T\right)$ for equilibrium constants, tabulated as a function of $\log _{10}(T)$, would provide results with relative accuracy better than $10^{-4}$ with the minimum number of points. This grid contained 31 points. We then constructed a temperature grid that was very close to the adaptive one, but using only round numbers, as well as adding some low temperature points to extend the grid down to $10^{-5} \mathrm{~K}$. This grid was then used for our final calculations, and contains 42 points. Partition functions and equilibrium constants for molecules are presented in Tables 6 and 7. Partition functions for atoms are presented in Table 8. The full tables are available at the CDS.

Partition functions for $\mathrm{H}_{2}$ and $\mathrm{CO}$, two cases of common astrophysical interest are examined in Fig. 3 and compared with previous results from ST and Irwin (1987), and in the case of CO with Gamache et al. (2000). The main impact of the new calculations is seen in the upper and middle panels: our calculations go down to near absolute zero temperature and there approach a constant value, specifically the statistical weight of the ground level divided by the nuclear statistical weight. The lower panels show the impact of inclusion of high-lying states, higher-order constants and changes to the molecular data compared to ST. We see in $\mathrm{H}_{2}$ the inclusion of higher-order constants has some effect at higher temperatures, while the addition of high-lying states has no appreciable effect. The updating of the spectroscopic constants according to Irikura (2007) has a very small effect on the partition function. In $\mathrm{CO}$, we see none of these has a significant effect.

Our partition functions for atoms and ions are overall in very good agreement with results from Irwin (1987) and Lawler \& Sneden (priv. comm.), where the temperature ranges considered by the various sets of calculations overlap. The Lawler \& Sneden data are the basis of the partition functions used in the MOOG spectral synthesis code (Sneden 1973 and updates). The original partition function data, kindly provided 
Table 2. Sample of table for $\mathrm{H}_{2}$ containing adopted dissociation energy, nuclear spins, and spectroscopic constants.

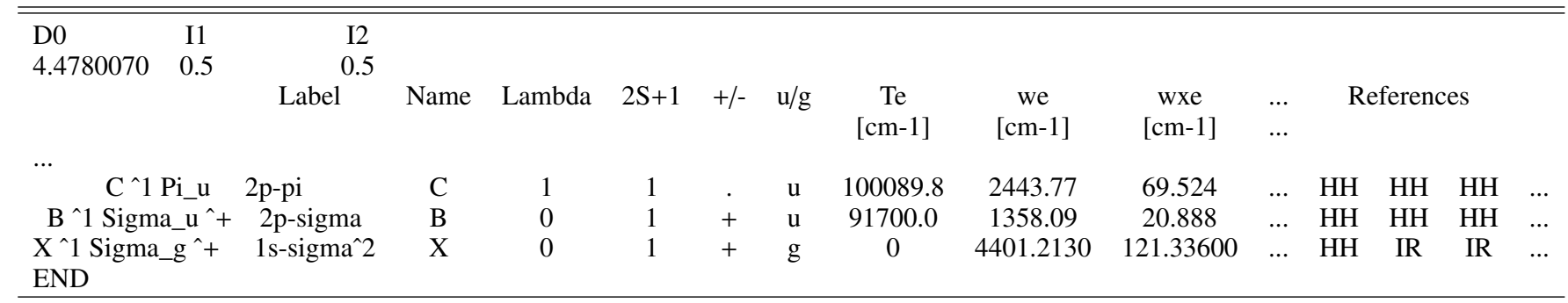

Notes. The full table is available at the CDS.

Table 3. Key to reference labels in electronic files. The full details of each are given in the reference list of this paper.

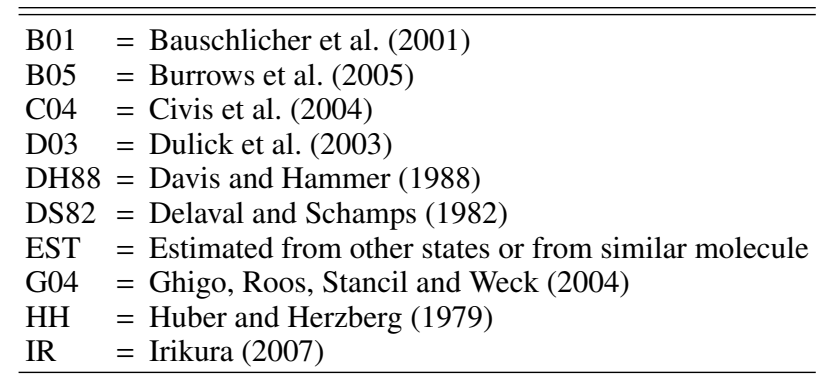

Table 4. Sample of table with first, second, and third ionisation energies for atoms and ions (for $\mathrm{H}$ and $\mathrm{He}$, the highest possible ionisation stages are singly and doubly ionised, respectively, and therefore the ionisation energy of higher stages are meaningless and are assigned a value of -1 in the table).

\begin{tabular}{ccccc}
\hline $\begin{array}{c}\text { * Atom. Num. } \\
*\end{array}$ & Elem. & $\begin{array}{c}\text { IE1 } \\
{[\mathrm{eV}]}\end{array}$ & $\begin{array}{c}\text { IE2 } \\
{[\mathrm{eV}]}\end{array}$ & $\begin{array}{c}\text { IE3 } \\
{[\mathrm{eV}]}\end{array}$ \\
\hline 1 & $\mathrm{H}$ & 13.5984 & -1.000 & -1.000 \\
2 & $\mathrm{He}$ & 24.5874 & 54.418 & -1.000 \\
3 & $\mathrm{Li}$ & 5.3917 & 75.640 & 122.454 \\
4 & $\mathrm{Be}$ & 9.3227 & 18.211 & 153.896 \\
5 & $\mathrm{~B}$ & 8.2980 & 25.155 & 37.931 \\
6 & $\mathrm{C}$ & 11.2603 & 24.385 & 47.888
\end{tabular}

Notes. The full table is available at the CDS.

to us by Lawler \& Sneden are in some cases also based on NIST data, e.g. Tb II (Lawler et al. 2001) and Ce II (Lawler et al. 2009), while for a few cases taken from other sources, e.g. Ho II taken from Bord \& Cowley (2002, see Lawler et al. 2004). These authors, however, provide polynomial fits or partition function data that are optimised or tabulated only for temperatures above $\sim 500-1000 \mathrm{~K}$ and below $\sim 10000-16000 \mathrm{~K}$ but are generally not applicable or available outside the designated range. For instance, the values of the polynomial fits provided by Irwin (1987) would diverge very rapidly for temperatures outside the indicated range and are therefore not suited for applications to low-temperature astrophysical environments. Lawler \& Sneden do provide data at $1 \mathrm{~K}$, but the $1-500 \mathrm{~K}$ range is generally not sampled (or is, at best, largely undersampled), which makes it difficult in practice to accurately interpolate the values of the partition functions for in-between temperatures. In Fig. 4, we present some examples of partition functions we calculated for various atoms and ions of astrophysical importance for molecule formation and stellar spectroscopy
Table 5. Sample of table with the list of references for atomic energy level data, ion by ion.

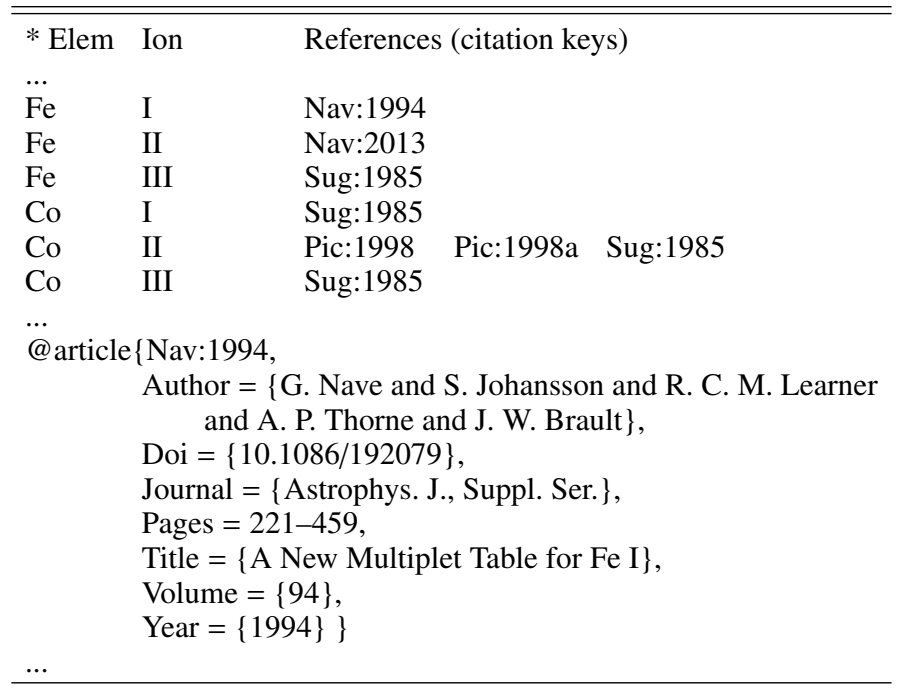

Notes. The citation keys in the table are abbreviations of the full references, which are also made available electronically in $\mathrm{BIBT}_{\mathrm{E}} \mathrm{X}$ format. The full table is available at the CDS.

and compare our results with the ones from Irwin (1987) and Lawler \& Sneden. Agreement is generally good where the respective temperature ranges of the various sources overlap $(T>$ $1000 \mathrm{~K}$ ), but information at lower temperatures are missing in the Irwin (1987) and Lawler \& Sneden data sets. Simple extrapolation or, where possible, interpolation thereof yields inaccurate results at low temperatures. Figure 5 shows an analogous comparison for some lanthanide ions of astrophysical interest for stellar spectroscopy of neutron-capture elements. Agreement among the various sources is less satisfactory here, with the Irwin (1987) data set showing the largest deviations with respect to our calculations and Lawler \& Sneden's. The last two are in excellent agreement with each other in this case, the only limitation of the Lawler \& Sneden data set being again the sparser sampling at low temperatures. The largest differences are found for Pm II and Pr II (Fig. 6) for which our partition functions at high temperatures are $\sim 20-30 \%$ lower than the Lawler \& Sneden ones.

As mentioned in Sect. 2, the summation over states in the partition function calculations for atoms and molecules is performed over all energy levels for which data are available. In the case of molecules, only a few cases such as $\mathrm{H}_{2}$ have a significant number of identified highly-excited states. As seen in Fig. 3 the exclusion of states above $40000 \mathrm{~cm}^{-1}$ has practically no effect 
Table 6. Sample of table containing partition functions for molecules.

\begin{tabular}{|c|c|c|c|c|c|c|c|c|}
\hline \multicolumn{9}{|c|}{$\begin{array}{c}\text { Partition functions Q } \\
291\end{array}$} \\
\hline $\mathrm{T}[\mathrm{K}]$ & $1.00000 \mathrm{e}-05$ & $1.00000 \mathrm{e}-04$ & $\ldots$ & $1.00000 \mathrm{e}+02$ & $\ldots$ & $5.00000 \mathrm{e}+03$ & $\ldots$ & $1.00000 \mathrm{e}+04$ \\
\hline $\mathrm{H} 2$ & $2.50000 \mathrm{e}-01$ & $2.50000 \mathrm{e}-01$ & $\cdots$ & $6.67129 \mathrm{e}-01$ & $\ldots$ & $5.07619 \mathrm{e}+01$ & $\ldots$ & $1.94871 \mathrm{e}+02$ \\
\hline Li2 & $3.75000 \mathrm{e}-01$ & $3.75000 \mathrm{e}-01$ & $\ldots$ & $5.26396 \mathrm{e}+01$ & $\ldots$ & $5.44859 e+04$ & $\ldots$ & $2.97872 \mathrm{e}+05$ \\
\hline B2 & $1.87500 \mathrm{e}+00$ & $1.87500 \mathrm{e}+00$ & $\ldots$ & $8.70213 e+01$ & $\ldots$ & $1.86115 \mathrm{e}+04$ & $\ldots$ & $8.32502 \mathrm{e}+04$ \\
\hline $\mathrm{CO}$ & $1.00000 \mathrm{e}+00$ & $1.00000 \mathrm{e}+00$ & $\ldots$ & $3.64899 \mathrm{e}+01$ & .. & $4.06593 e+03$ & $\begin{array}{l}\cdots \\
\cdots\end{array}$ & $1.50689 \mathrm{e}+04$ \\
\hline KI & $1.00000 \mathrm{e}+00$ & $1.00000 \mathrm{e}+00$ & $\ldots$ & $1.23175 \mathrm{e}+03$ & $\begin{array}{l}\ldots \\
\ldots\end{array}$ & $1.57038 \mathrm{e}+06$ & $\begin{array}{l}\ldots \\
\ldots\end{array}$ & $8.03200 \mathrm{e}+06$ \\
\hline
\end{tabular}

Notes. The full table is available at the CDS.

Table 7. Sample of table containing equilibrium constants.

\begin{tabular}{|c|c|c|c|c|c|c|c|c|}
\hline \multicolumn{9}{|c|}{ Equilibrium constants $\log 10(\mathrm{pK})$} \\
\hline & 291 & & & & & & & \\
\hline $\mathrm{T}[\mathrm{K}]$ & $1.00000 \mathrm{e}-05$ & $1.00000 \mathrm{e}-04$ & $\ldots$ & $1.00000 \mathrm{e}+02$ & $\ldots$ & $5.00000 \mathrm{e}+03$ & $\ldots$ & $1.00000 \mathrm{e}+04$ \\
\hline $\mathrm{H} 2$ & $-2.25687 e+09$ & $-2.25687 e+08$ & $\ldots$ & $-2.16942 \mathrm{e}+02$ & $\ldots$ & $6.59790 \mathrm{e}+00$ & $\ldots$ & $9.02320 \mathrm{e}+00$ \\
\hline Li2 & $-5.29139 \mathrm{e}+08$ & $-5.29139 e+07$ & $\ldots$ & $-4.48086 e+01$ & $\ldots$ & $8.31687 \mathrm{e}+00$ & $\ldots$ & $9.39624 \mathrm{e}+00$ \\
\hline B2 & $-1.41218 \mathrm{e}+09$ & $-1.41218 \mathrm{e}+08$ & $\ldots$ & $-1.32211 \mathrm{e}+02$ & $\cdots$ & $8.22075 \mathrm{e}+00$ & $\ldots$ & $9.77654 \mathrm{e}+00$ \\
\hline $\mathrm{CO}$ & $-5.60286 e+09$ & $-5.60286 e+08$ & $\cdots$ & $-5.50670 \mathrm{e}+02$ & $\begin{array}{l}\cdots \\
\cdots\end{array}$ & $1.01177 \mathrm{e}+00$ & $\cdots$ & $6.87062 \mathrm{e}+00$ \\
\hline KI & $-1.66317 \mathrm{e}+09$ & $-1.66317 \mathrm{e}+08$ & $\cdots$ & $-1.57877 \mathrm{e}+02$ & $\ldots$ & $6.32569 \mathrm{e}+00$ & $\ldots$ & $8.72302 \mathrm{e}+00$ \\
\hline
\end{tabular}

Notes. The full table is available at the CDS.

Table 8. Sample of table containing partition functions for atoms.

\begin{tabular}{|c|c|c|c|c|c|c|c|c|}
\hline \multicolumn{9}{|c|}{ Partition functions Q } \\
\hline $\mathrm{T}[\mathrm{K}]$ & $1.00000 \mathrm{e}-05$ & $1.00000 \mathrm{e}-04$ & $\ldots$ & $1.00000 \mathrm{e}+02$ & $\ldots$ & $5.00000 \mathrm{e}+03$ & $\ldots$ & $1.00000 \mathrm{e}+04$ \\
\hline H_I & $2.00000 \mathrm{e}+00$ & $2.00000 \mathrm{e}+00$ & $\ldots$ & $2.00000 \mathrm{e}+00$ & $\ldots$ & $2.00000 \mathrm{e}+00$ & $\ldots$ & $2.00015 \mathrm{e}+00$ \\
\hline H_II & $1.00000 \mathrm{e}+00$ & $1.00000 \mathrm{e}+00$ & $\ldots$ & $1.00000 \mathrm{e}+00$ & $\ldots$ & $1.00000 \mathrm{e}+00$ & $\ldots$ & $1.00000 \mathrm{e}+00$ \\
\hline D_I & $2.00000 \mathrm{e}+00$ & $2.00000 \mathrm{e}+00$ & $\ldots$ & $2.00000 \mathrm{e}+00$ & $\ldots$ & $2.00000 \mathrm{e}+00$ & $\ldots$ & $2.00014 \mathrm{e}+00$ \\
\hline $\mathrm{Fe} \_\mathrm{I}$ & $9.00000 \mathrm{e}+00$ & $9.00000 \mathrm{e}+00$ & $\begin{array}{l}\cdots \\
\cdots\end{array}$ & $9.01785 \mathrm{e}+00$ & $\begin{array}{c}\cdots \\
\ldots\end{array}$ & $2.77940 \mathrm{e}+01$ & $\begin{array}{l}\cdots \\
\cdots\end{array}$ & $5.96627 \mathrm{e}+01$ \\
\hline $\mathrm{Cl}-$ & $1.00000 \mathrm{e}+00$ & $1.00000 \mathrm{e}+00$ & $\begin{array}{l}\cdots \\
\cdots\end{array}$ & $1.00000 \mathrm{e}+00$ & $\begin{array}{l}\cdots \\
\ldots\end{array}$ & $1.00000 \mathrm{e}+00$ & $\begin{array}{l}\cdots \\
\ldots\end{array}$ & $1.00000 \mathrm{e}+00$ \\
\hline
\end{tabular}

Notes. The full table is available at the CDS.

on the partition function. The most likely scenario for molecules is that missing high-lying states leads to an underestimate of the partition function, leading to an overestimate of the equilibrium constant. For cases such as $\mathrm{H}_{2}$ this error can be expected to be small at all temperatures, but in cases where few excited states are identified this may lead to significant errors particularly at higher temperatures.

In the case of atoms, NIST often provides a very large number of Rydberg states. Hummer \& Mihalas (1988) investigated the sensitivity of a hydrogenic partition function to truncation of the summation at a given cutoff effective principal quantum number $n^{*}$. They showed that sensitivity to the choice of cutoff is dependent on $I / k T$, where $I$ is the ionisation potential of the species of interest. In particular, when $I / k T$ is relatively large, say greater than 10 , the ground state dominates the partition function, and the result is quite insensitive for any reasonable choice of the cutoff. However, as $I / k T$ becomes smaller the partition function becomes sensitive to the cutoff. Thus, at any given temperature we expect species with low ionisation potentials (i.e. neutral species of alkalis and alkaline earths) to be the most sensitive to the cutoff procedure, and for any given species the sensitivity increases with temperature.

To investigate this we performed calculations in the chemical picture using the occupation probability formalism (Hummer \& Mihalas 1988). The occupation probability defines the likelihood that a given state is not dissolved by the perturbations of nearby particles. We calculate this probability for conditions approximating three depths in the solar atmosphere, using Eq. (4.71) of Hummer \& Mihalas (1988), where the part relating to perturbations by charged particles is replaced with the expressions from Appendix A of Hubeny et al. (1994). The results are shown in Fig. 7, and indicate that in the typical line forming regions of a solar-type stellar atmosphere, states with $n^{*} \gtrsim 12$ will be dissolved and these states should be excluded from the partition function summation. We performed calculations for a significant number of elements where a cutoff was 

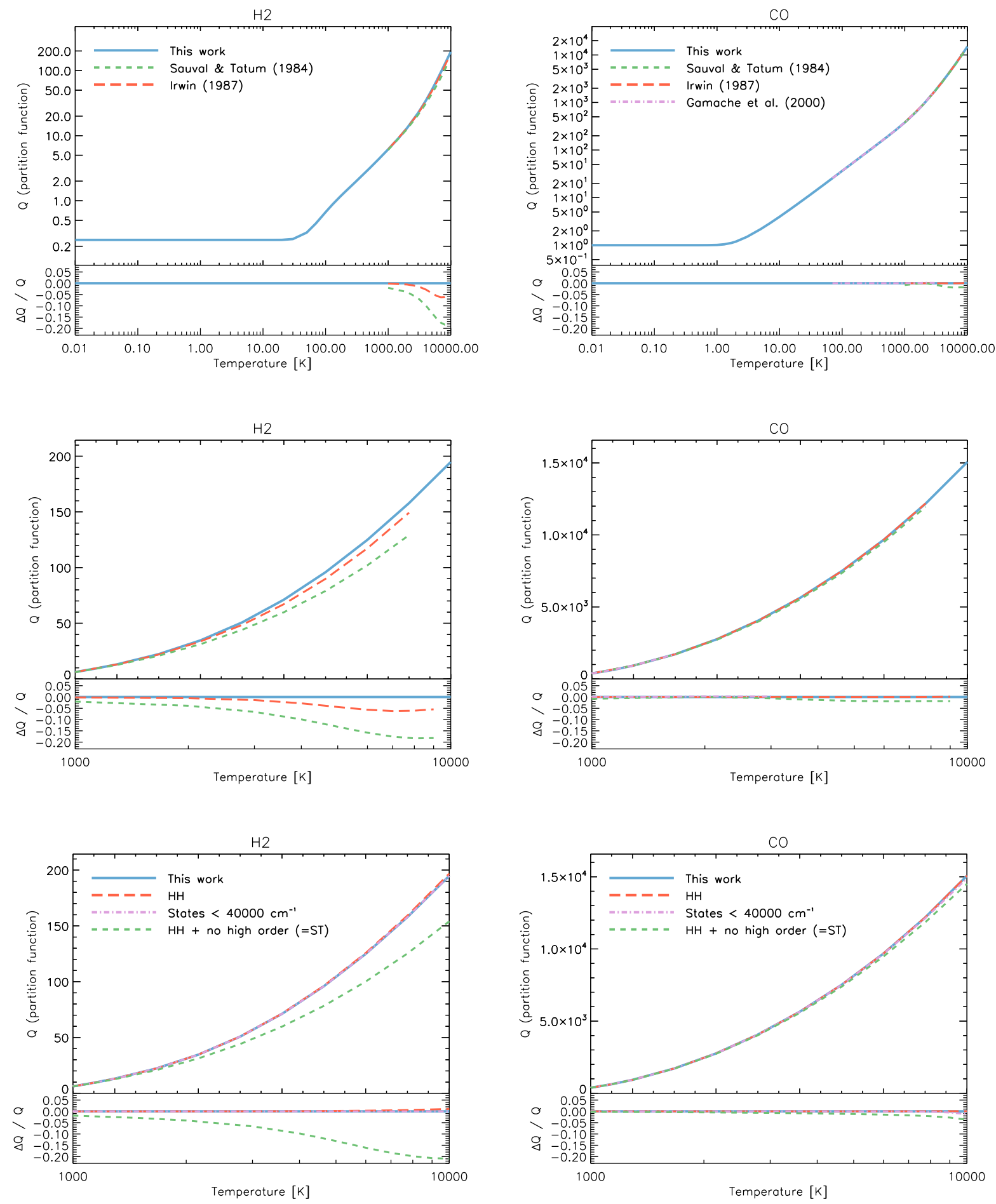

Fig. 3. Partition function comparisons for $\mathrm{H}_{2}$ (left column) and $\mathrm{CO}$ (right column). Upper row: partition function $(Q)$ calculated in this work (blue curves) shown on a logarithmic scale. Results from polynomial expressions of ST (green dashed curves), Irwin (1987; red long-dashed curves), and Gamache et al. (2000; purple dot-dashed curves) are also shown. Middle row: same results as for the upper panels are shown on a linear scale over the reduced temperature range 1000 to $10000 \mathrm{~K}$. Lower row: partition functions calculated in this work under various approximations, allowing the origin of any changes with respect to ST to be identified. Partition functions are recalculated assuming constants from HH, i.e., changes and additions to molecular data are ignored (red long-dashed curves), limiting states to those with $T_{e}<40000 \mathrm{~cm}^{-1}$, as was done in ST, and thus showing the contribution of high-lying states (purple dot-dashed curves), and with both of these approximations (neglecting highlying states and using only data from $\mathrm{HH}$ ), and in addition neglecting higher-order spectroscopic constants that were not considered by ST (green dashed curves). This final calculation should correspond to that of ST (which it does to reasonable precision by comparison with the middle panel), and allows the effect of the higher-order constants to be seen. In each case, the lower panels show relative differences between the plotted results and ours. 
P. S. Barklem and R. Collet: Partition functions for molecules and atoms

C I

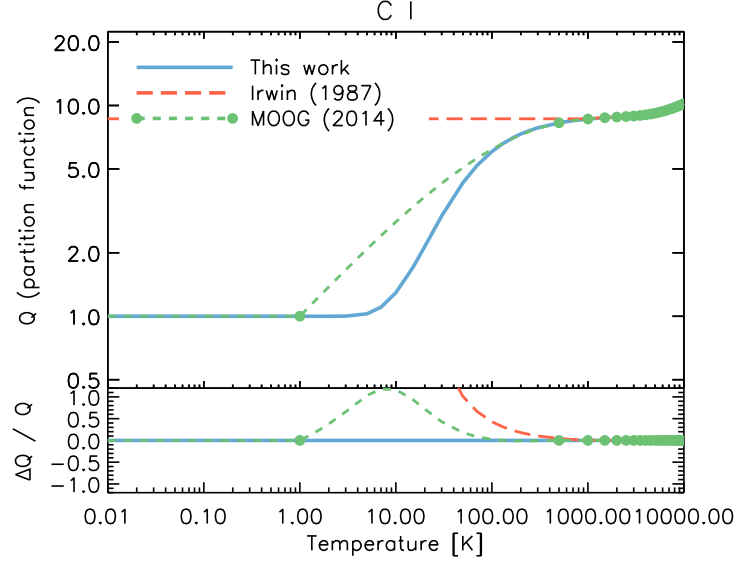

0
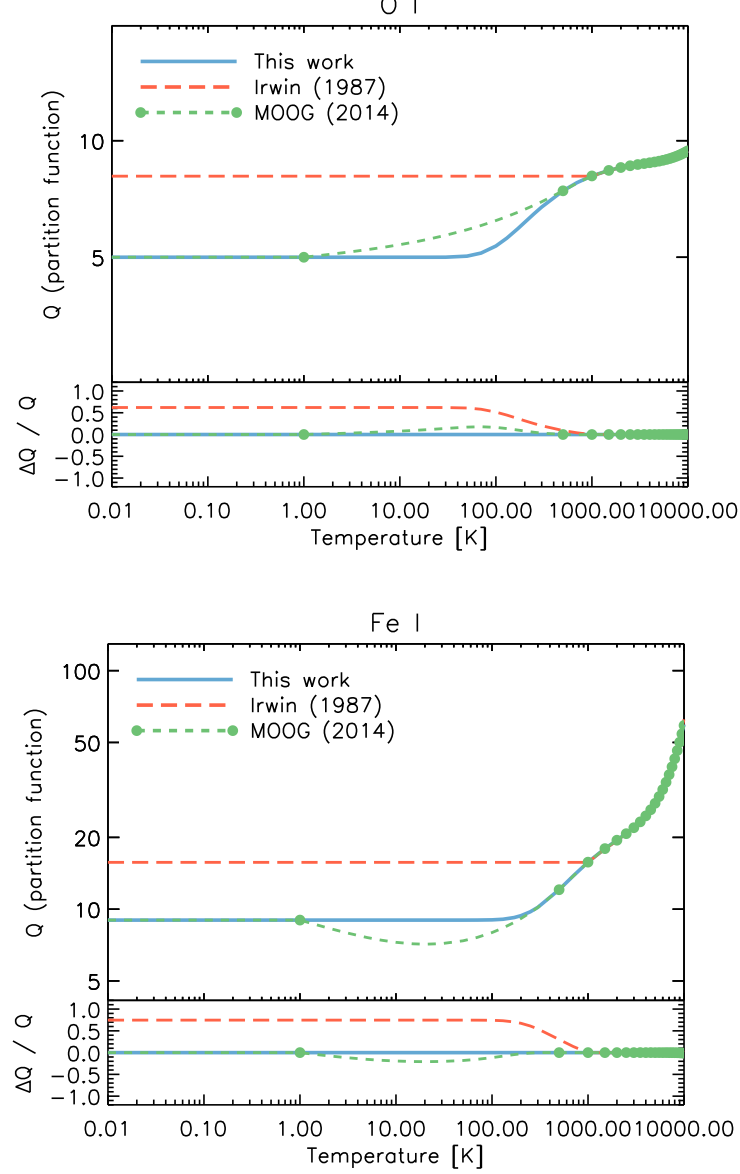

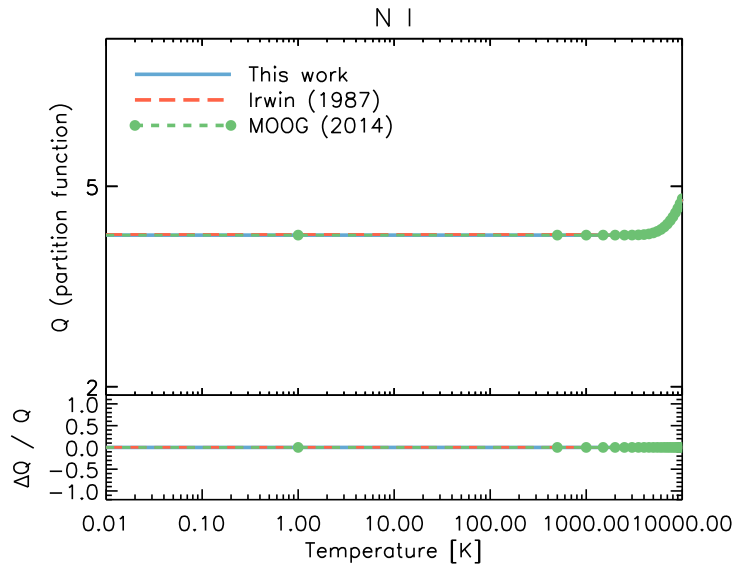

Si 1
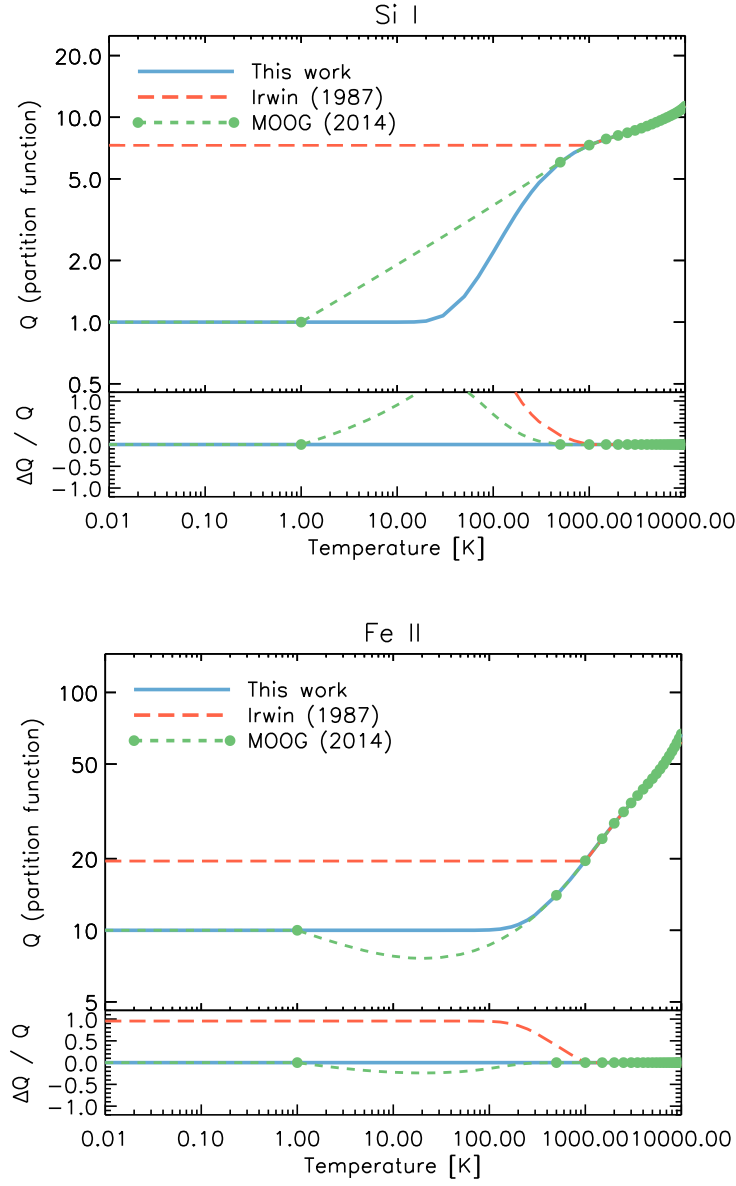

Fig. 4. Upper panels: comparison between the atomic partition functions (Q) calculated in this work with data from NIST (blue curves) and the ones provided by Irwin (1987; red curves) and by Lawler \& Sneden (MOOG data, version from July 2014; see also Sneden 1973 and updates; green dots). Partition functions from Irwin (1987) are extended to temperatures below $1000 \mathrm{~K}$ using constant extrapolation (red dashed curves). Data from Lawler \& Sneden are interpolated in between the sampled temperature points using cubic splines in $\log Q$. Lower panels: relative differences between the partition function calculations by the other authors and ours.

implemented for values between $n^{*}=10$ and 20. For neutral species of many atoms of astrophysical interest, such as $\mathrm{C}, \mathrm{O}$, $\mathrm{Mg}$, and $\mathrm{Fe}$, and for all ionised species, the results showed no significant changes compared to the final results including all states across the entire temperature range; much less than the per cent level. However, as expected, neutral species of alkalis, which have low ionisation potentials, showed some effects at higher temperatures; see Fig. 8. At temperatures corresponding to the line forming regions of solar-type stars, around 5000$6000 \mathrm{~K}$, which indeed form lines of neutral alkalis, we see that differences for a cutoff of $n^{*} \approx 12$ are of order a couple of per cent. This indicates that in such cases, the partition function calculated here would be overestimated, and if greater accuracy is required, the plasma conditions must be accounted for. We note that the effects are even larger at higher temperatures, but very few neutral atoms will be found at these temperatures, except at high densities where plasma interactions must be accounted for. In general these results are not appropriate for environments with densities much greater than those typical of stellar atmospheres. 

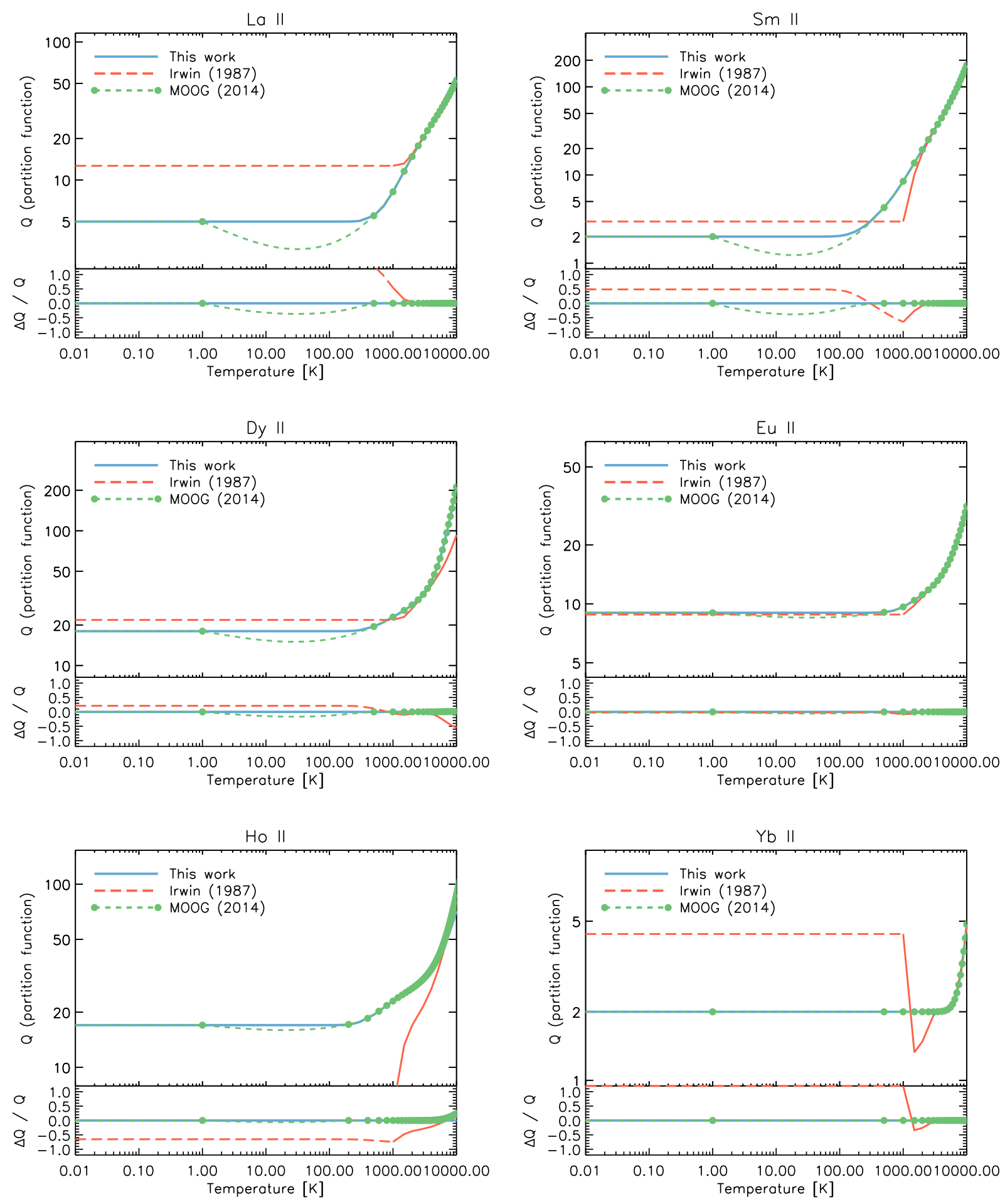

Fig. 5. Similar to Fig. 4, for some of the lanthanide ions. Note, the Yb II partition function from polynomial fits from Irwin (1987) shows an unphysical sharp decrease between 1000 and $2000 \mathrm{~K}$.

\section{Concluding remarks}

Calculations of partition functions and equilibrium constants for 291 diatomic molecules have been presented, and dissociation energy data collated for these molecules. The calculations are based on spectroscopic constants from $\mathrm{HH}$, with updates to constants for the ground states of 85 molecules by Irikura (2007, 2009), covering the most important molecules. We note that there are other compilations containing partition function data for some diatomic molecules. For example Gamache et al. (2000) for $\mathrm{CO}, \mathrm{O}_{2}, \mathrm{NO}, \mathrm{OH}, \mathrm{HF}, \mathrm{HCl}, \mathrm{HBr}, \mathrm{HI}, \mathrm{ClO}, \mathrm{N}_{2}$, and $\mathrm{NO}^{+}$, over the temperature range 70 to $3005 \mathrm{~K}$, and Babou et al. (2009) for $\mathrm{N}_{2}, \mathrm{~N}_{2}^{+}, \mathrm{NO}, \mathrm{O}_{2}, \mathrm{CN}, \mathrm{C}_{2}, \mathrm{CO}$, and $\mathrm{CO}^{+}$, up to $50000 \mathrm{~K}$, though these data are only available on request. We note that all but two of these molecules are covered by the updates from Irikura; the exceptions being $\mathrm{HBr}$, which is 
P. S. Barklem and R. Collet: Partition functions for molecules and atoms
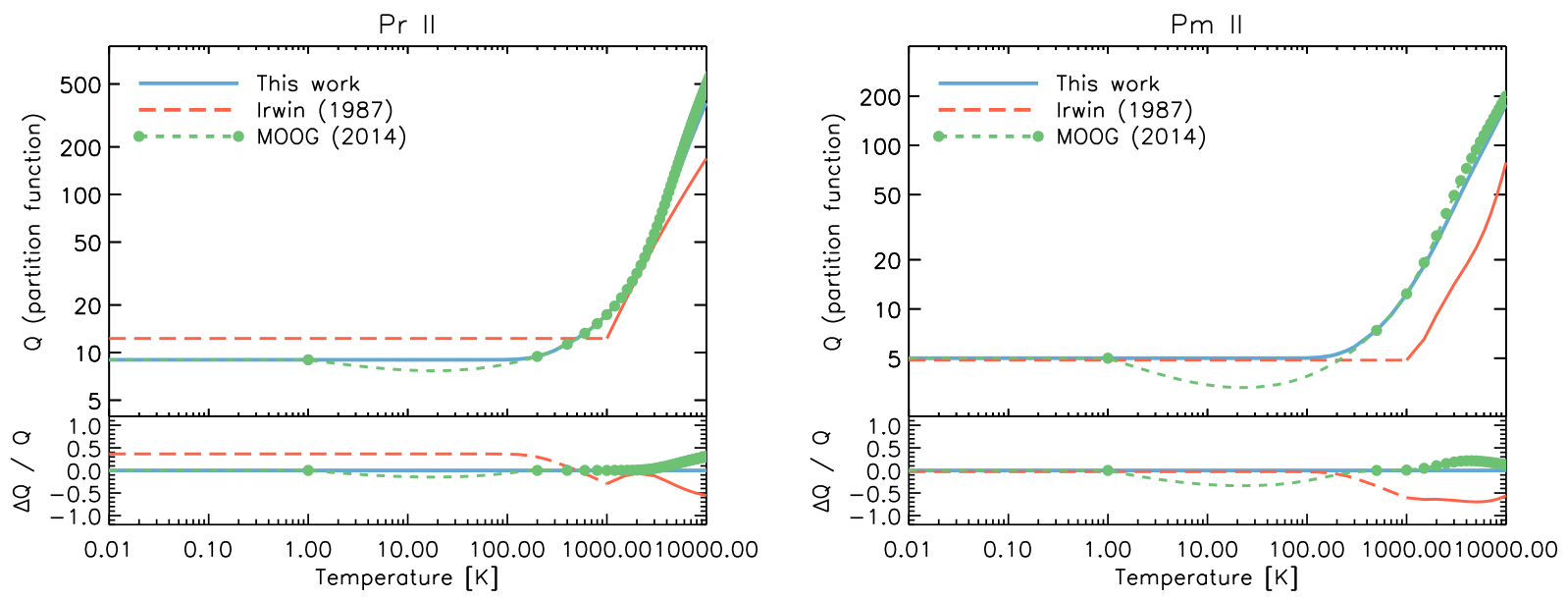

Fig. 6. Similar to Figs. 4 and 5, for Pr II and Pm II, which, among the lanthanide ions, show the largest differences in terms of partition function values between our NIST-based calculations (blue curves) and Lawler \& Sneden's (green dots) data at temperatures below $10000 \mathrm{~K}$.

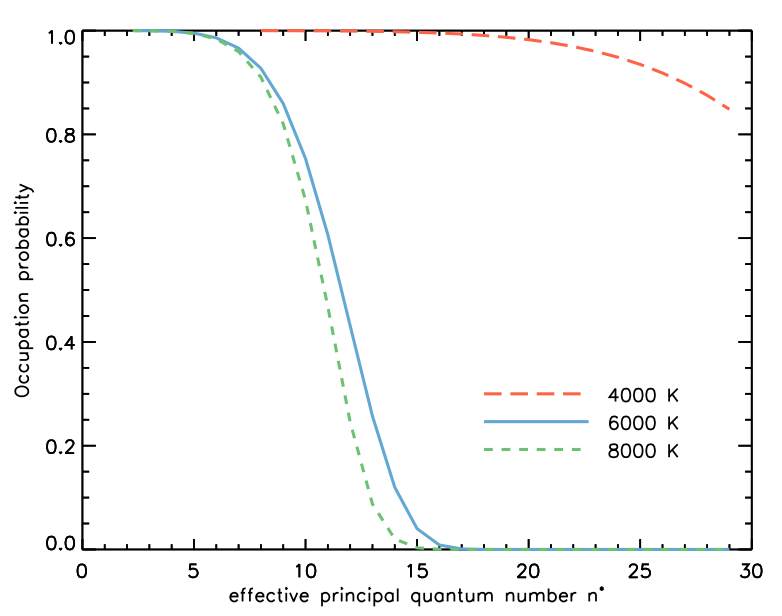

Fig. 7. Occupation probability as a function of effective principal quantum number $n^{*}$ for conditions representative of three depths in the solar atmosphere. The depths are labelled by their approximate temperatures, and these correspond to particle number densities of $N_{\mathrm{H}}$ and $N_{\mathrm{e}}$, of $10^{14}$ and $10^{13} \mathrm{~cm}^{-3}$ at $4000 \mathrm{~K}$, of $10^{17}$ and $3 \times 10^{13} \mathrm{~cm}^{-3}$ at $6000 \mathrm{~K}$, and of $10^{17}$ and $7 \times 10^{14} \mathrm{~cm}^{-3}$ at $8000 \mathrm{~K}$, respectively. In each case, $N_{\mathrm{He}}=0.1 \times N_{\mathrm{H}}$.

not covered by our study, and HI, which is probably of limited astrophysical importance. Thus, we expect that the calculations presented here for these cases are of similar accuracies to these studies. For example, in the important case of $\mathrm{CO}$, we note that the spectroscopic constants employed by us and those employed by Babou et al. (2009) for the ground state agree in all cases to better than four significant figures, and it was seen in Fig. 3 that the partition functions agree almost exactly. We also note that there are numerous single molecule studies, e.g. Stancil (1996), Engel et al. (2005), often based on energy levels derived from explicit solution of the Schrödinger equation in quantum chemistry potentials. Merging these results into our results would be non-trivial, primarily due to differences in the temperature ranges. We emphasise that if a specific molecule of interest is important it may be worth to compare the results from these calculations with those from single molecule studies, and perhaps replace the data.
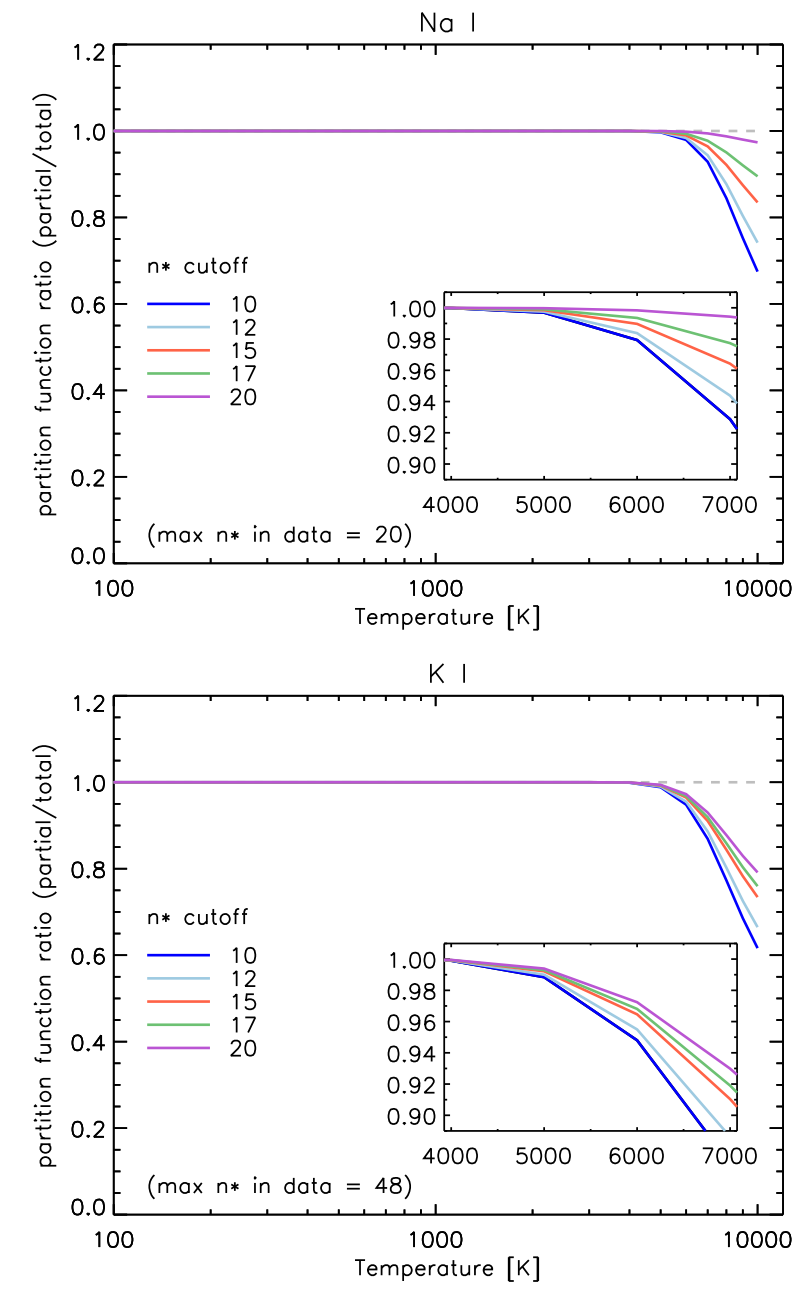

Fig. 8. Ratio of partial partition functions calculated with a $n^{*}$ cutoff in the summation between 10 and 20 , to the partition function calculated in this work including all states from NIST.

Partition functions have also been calculated for 284 species of atoms for all elements from $\mathrm{H}$ to $\mathrm{U}$. The data are based on up-to-date NIST critically compiled data. We have investigated the possible influence of inclusion of many Rydberg states, and 
estimate that this should not lead to uncertainties larger than a couple of per cent in typical applications. If higher accuracy is required, then more sophisticated methods allowing for interactions with the environment may be required.

Finally, we comment that critical compilation of partition functions and/or the data necessary to calculate them has become a task that is probably beyond any individual or even small group. It seems to us that the most reasonable approach is a distributed one, where the community builds a definitive set of partition functions and spectroscopic constants and/or potentials and/or energy levels, updated via gradual improvements from studies of individual or small sets of molecules. This would require some kind of centralised infrastructure in the form of a database or similar, and would be important for both the astrophysics and molecular physics communities.

Acknowledgements. We are grateful to Jim Lawler and Chris Sneden for supplying their raw partition function data for atoms and ions. We thank Nikolai Piskunov for stimulation of, and interest in, this project and support for visits by R.C. to Uppsala. We are thankful to Alexander Kramida from NIST for assisting us with the compilation of the list of sources for atomic energy level data. We gratefully acknowledge the support of the Royal Swedish Academy of Sciences, Göran Gustafssons Stiftelse and the Swedish Research Council. P.S.B. was a Royal Swedish Academy of Sciences Research Fellow supported by a grant from the Knut and Alice Wallenberg Foundation during much of this work. P.S.B. is also supported by the project grant "The New Milky" from the Knut and Alice Wallenberg foundation. R.C. acknowledges support from the Australian Research Council through a Discovery Early Career Researcher Award (DECRA) grant (project DE120102940).

\section{References}

Babou, Y., Rivière, P., Perrin, M.-Y., \& Soufiani, A. 2009, Int. J. Thermophys., 30, 416

Bauschlicher, C. W., Ram, R. S., Bernath, P. F., Parsons, C. G., \& Galehouse, D. 2001, J. Chem. Phys., 115, 1312

Bernath, P. F. 2009, Int. Rev. Phys. Chem., 28, 681

Bond, J. C., O’Brien, D. P., \& Lauretta, D. S. 2010, ApJ, 715, 1050

Bord, D. J., \& Cowley, C. R. 2002, Sol. Phys., 211, 3

Burrows, A., Dulick, M., Bauschlicher, Jr., C. W., et al. 2005, ApJ, 624, 988

Civiš, S., Šebera, J., Špirko, V., et al. 2004, J. Mol. Struct., 695, 5
Costes, M., \& Naulin, C. 1994, in Molecules in the Stellar Environment, ed. U. G. Jorgensen, IAU Colloq. 146 (Berlin: Springer Verlag), Lect. Notes Phys., 428, 250

Curtiss, L. A., Raghavachari, K., Trucks, G. W., \& Pople, J. A. 1991, J. Chem. Phys., 94, 7221

Däppen, W., Anderson, L., \& Mihalas, D. 1987, ApJ, 319, 195

Davis, S. P., \& Hammer, P. D. 1988, ApJ, 332, 1090

Delaval, J. M., Dufour, C., \& Schamps, J. 1980, J. Phys. B: Atom. Mol. Phys., 13,4757

Dulick, M., Bauschlicher, Jr., C. W., Burrows, A., et al. 2003, ApJ, 594, 651

Engel, E. A., Doss, N., Harris, G. J., \& Tennyson, J. 2005, MNRAS, 357, 471

Gamache, R. R., Kennedy, S., Hawkins, R. L., \& Rothman, L. S. 2000, J. Mol. Struct., 517, 407

Ghigo, G., Roos, B. O., Stancil, P. C., \& Weck, P. F. 2004, J. Chem. Phys., 121, 8194

Haynes, W. M. 2010, CRC Handbook of Chemistry and Physics, 91st edn. (CRC Press, Taylor and Francis Group)

Herzberg, G. 1950, Molecular spectra and molecular structure, Spectra of diatomic molecules, Vol. 1

Hubeny, I., Hummer, D. G., \& Lanz, T. 1994, A\&A, 282, 151

Huber, K. P., \& Herzberg, G. 1979, Constants of Diatomic Molecules (Van Nostrand Reinhold)

Huber, K. P., Herzberg, G., Gallagher, J. W., \& Johnson III, R. D. 2011 NIST Chemistry WebBook, NIST Standard Reference Database Number 69 (Gaithersburg MD: National Institute of Standards and Technology), 20899 http: //webbook.nist.gov, retrieved October 14

Hummer, D. G., \& Mihalas, D. 1988, ApJ, 331, 794

Irikura, K. K. 2007, J. Phys. Chem. Ref. Data, 36, 389

Irikura, K. K. 2009, J. Phys. Chem. Ref. Data, 38, 749

Irwin, A. W. 1987, A\&A, 182, 348

Kramida, A., Ralchenko, Y., Reader, J., \& NIST ASD Team. 2014, NIST Atomic Spectra Database (ver. 5.2), 2015 (Gaithersburg, MD: National Institute of Standards and Technology), http://physics.nist.gov/asd, June 23

Lawler, J. E., Sneden, C., \& Cowan, J. J. 2004, ApJ, 604, 850

Lawler, J. E., Wickliffe, M. E., Cowley, C. R., \& Sneden, C. 2001, ApJS, 137, 341

Lawler, J. E., Sneden, C., Cowan, J. J., Ivans, I. I., \& Hartog, E. A. D. 2009, ApJS, 182, 51

Luo, Y.-R. 2007, Comprehensive Handbook of Chemical Bond Energies (CRC Press)

Rogers, F. J., Swenson, F. J., \& Iglesias, C. A. 1996, ApJ, 456, 902

Sauval, A. J., \& Tatum, J. B. 1984, ApJS, 56, 193

Sneden, C. 1973, ApJ, 184, 839

Stancil, P. C. 1996, J. Quant. Spectr. Rad. Transf., 55, 849

Tatum, J. B. 1966, Publications of the Dominion Astrophysical Observatory Victoria, 13, 1 